\title{
Comparison of fast ion collective Thomson scattering measurements at ASDEX Upgrade with numerical simulations
}

Salewski, Mirko; Meo, Fernando; Stejner Pedersen, Morten; Asunta, O.; Bindslev, Henrik; Furtula, Vedran; Korsholm, Søren Bang; Kurki-Suonio, T.; Leipold, Frank; Leuterer, F.

Total number of authors:

17

Published in:

Nuclear Fusion

Link to article, DOI:

$10.1088 / 0029-5515 / 50 / 3 / 035012$

Publication date:

2010

Link back to DTU Orbit

Citation (APA):

Salewski, M., Meo, F., Stejner Pedersen, M., Asunta, O., Bindslev, H., Furtula, V., Korsholm, S. B., KurkiSuonio, T., Leipold, F., Leuterer, F., Michelsen, P., Moseev, D., Nielsen, S. K., Stober, J., Tardini, G., Wagner, D., \& Woskov, P. (2010). Comparison of fast ion collective Thomson scattering measurements at ASDEX Upgrade with numerical simulations. Nuclear Fusion, 50(3), 035012. https://doi.org/10.1088/0029$5515 / 50 / 3 / 035012$

\section{General rights}

Copyright and moral rights for the publications made accessible in the public portal are retained by the authors and/or other copyright owners and it is a condition of accessing publications that users recognise and abide by the legal requirements associated with these rights.

- Users may download and print one copy of any publication from the public portal for the purpose of private study or research.

- You may not further distribute the material or use it for any profit-making activity or commercial gain

- You may freely distribute the URL identifying the publication in the public portal 


\title{
Comparison of fast ion collective Thomson scattering measurements at ASDEX Upgrade with numerical simulations
}

\author{
M Salewski ${ }^{1}$, F Meo ${ }^{1}$, M Stejner ${ }^{1}$, O Asunta ${ }^{2}$, H Bindslev ${ }^{1}$, \\ V Furtula $^{1}$, S B Korsholm ${ }^{1}$, T Kurki-Suonio ${ }^{2}$, F Leipold $^{1}$, \\ F Leuterer ${ }^{3}$, P K Michelsen ${ }^{1}$, D Moseev ${ }^{1}$, S K Nielsen ${ }^{1}$, \\ J Stober $^{3}$, G Tardini $^{3}$, D Wagner ${ }^{3}, \mathbf{P}$ Woskov $^{4}$, and the \\ ASDEX Upgrade team ${ }^{3}$ \\ ${ }^{1}$ Association Euratom - Ris $\varnothing$ National Laboratory for Sustainable Energy, Technical \\ University of Denmark, DK-4000 Roskilde, Denmark \\ ${ }^{2}$ Association Euratom - TEKES, Helsinki University of Technology, FI-02015 TKK, \\ Finland \\ ${ }^{3}$ Max-Planck-Institut für Plasmaphysik, D-85748 Garching, Germany, Euratom \\ Association \\ ${ }^{4}$ MIT Plasma Science and Fusion Center, Cambridge, MA 02139, USA \\ E-mail: msal@risoe.dtu.dk
}

\begin{abstract}
Collective Thomson scattering (CTS) experiments were carried out at ASDEX Upgrade to measure the one-dimensional velocity distribution functions of fast ion populations. These measurements are compared with simulations using the codes TRANSP/NUBEAM and ASCOT for two different neutral beam injection (NBI) configurations: two NBI sources and only one NBI source. The measured CTS spectra as well as the inferred one-dimensional fast ion velocity distribution functions are clearly asymmetric as a consequence of the anisotropy of the beam ion populations and the selected geometry of the experiment. As expected, the one-beam configuration can clearly be distinguished from the two-beam configuration. The fast ion population is smaller and the asymmetry is less pronounced for the one-beam configuration. Salient features of the numerical simulation results agree with the CTS measurements while quantitative discrepancies in absolute values and gradients are found.
\end{abstract}

PACS numbers: 52.25.Os, 52.40.Db, 52.50.Gj, 52.65.Cc, 52.70.Gw 


\section{Introduction}

Medium-sized tokamaks such as ASDEX Upgrade are designed to confine hot plasmas with temperatures in the $\mathrm{keV}$ range and are usually equipped with powerful auxiliary heating systems: neutral beam injection (NBI), ion cyclotron resonance heating (ICRH) or electron cyclotron resonance heating (ECRH) [1,2]. The energetic ions generated by NBI and ICRH play a key role in heating the bulk plasma, and understanding fast ion physics is therefore essential. However, reliable predictions of fast ion motion still face many challenges: fast ions exhibit collective behavior [3,4] and can interact with MHD activity such as the family of Alfvén eigenmodes [5-8], sawteeth [9-12], kinetic ballooning modes $[6,13,14]$, neoclassical tearing modes $[6,15]$ or the sierpes mode [16]. Fast ions may also interact with turbulent fluctuations [17-19]. Theories describing such phenomena need to be tested against experimental data. The implications for ITER and tokamaks beyond are also far-reaching: the confinement of the bulk plasma and the fast ions may degrade due to such effects. Measurements of fast ion distributions have therefore been recognized as a milestone towards understanding plasmas in the burning regime [20,21]. Millimeter wave collective Thomson scattering (CTS) has been demonstrated to provide measurements of one-dimensional velocity distribution functions of confined fast ions in various selected directions and locations in the plasma at JET [22], TEXTOR [23,24] and ASDEX Upgrade [25, 26]; a fast ion CTS system at ITER is also foreseen [27-32]. CTS experiments at other machines have further contributed to the experience with millimeter wave CTS diagnostics [33-36]. For example, the ion temperature has been estimated by millimeter wave CTS at the W7-AS stellarator [37].

Here we report recent fast ion velocity distribution results obtained by CTS for different NBI configurations at ASDEX Upgrade. We compare the measurements with numerical simulations and describe methods for such a comparison. The experimental conditions are summarized in Section 2 and the methods in Section 3. The measured spectral power densities of scattered radiation are compared with synthetic CTS spectra calculated with a scattering model $[38,39]$ using fast ion velocity distributions obtained with the simulation codes TRANSP/NUBEAM [40,41] or ASCOT [42,43] (Section 4.1). Moreover, the inferred one-dimensional fast ion velocity distributions obtained for this experiment are presented and compared with the simulations (Section 4.2). We discuss perspectives and draw conclusions in Sections 5 and 6 .

\section{CTS experiment at ASDEX Upgrade}

\subsection{Plasma parameters}

The CTS measurements were carried out in a co-current NBI heated, standard H-mode plasma (discharge 24089). ASDEX Upgrade is equipped with two beam boxes each containing four neutral beam injectors [44]. We used two of the injectors, namely source 3 (S3) and source 8 (S8). S3 and S8 have full acceleration energies of $60 \mathrm{keV}$ and 


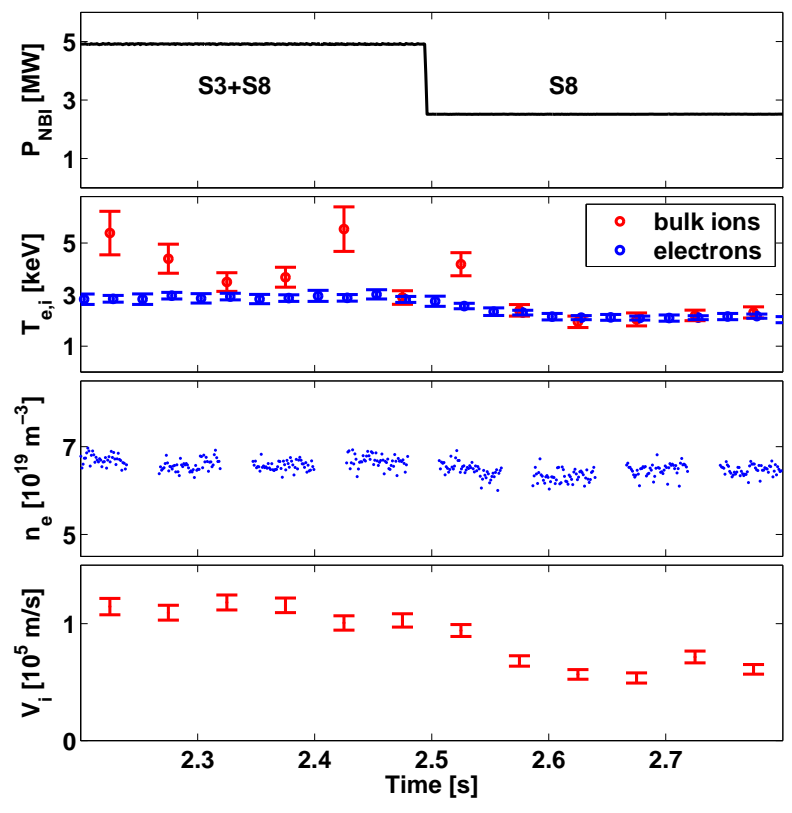

Figure 1. Time traces of NBI power, ion and electron temperatures, electron density and toroidal bulk ion drift velocity in the plasma center in discharge 24089. Neutral beams S3 and S8 heated the plasma up to $2.5 \mathrm{~s}$ when beam S3 was switched off.

$93 \mathrm{keV}$ in deuterium, respectively, and they have similar injection geometries. Both NBI sources $(\mathrm{S} 3+\mathrm{S} 8)$ injected deuterium with a total power of about $5 \mathrm{MW}(2 \times 2.5 \mathrm{MW})$, until S3 was switched off. Fast ion populations in the plasma center for both NBI configurations (S3+S8 and S8 only) were measured by CTS.

CTS spectra of scattered radiation depend not only on the fast ion velocity distribution function - the parameters of interest - but also on other parameters which are not the goal of the measurement and encumber the data analysis - the nuisance parameters. Among the nuisance parameters are several bulk plasma parameters which we discuss here. Figure 1 shows time traces of the NBI power, the electron and ion temperatures, the electron density and the toroidal bulk ion drift velocity. The temperatures and the drift velocity decreased when beam S3 was turned off whereas the electron density was feedback controlled. From here on we shall use representative values to characterize the plasma for both NBI configurations: two beams (S3+S8) versus one beam (S8). These representative values and their $1 \sigma$ confidence levels are given in Table 1 which also contains additional plasma parameters affecting the CTS spectra: the magnetic field, the isotope ratio and impurity concentrations. The ion temperature and bulk ion drift velocity were obtained from the charge exchange diagnostic, the electron temperature from (non-collective) Thomson scattering [45], the electron density from the integrated data analysis (IDA) [46-49], the isotope ratio from a neutral particle analyzer, and the impurity content was estimated by spectroscopy [50]. 
Table 1. Parameters of the plasma in discharge 24089 at the location of the CTS scattering volume $\left(\rho_{\text {pol }}=0\right)$ and their estimated $1 \sigma$ uncertainties. The parameters are given separately for the NBI configurations with two beams (S3+S8) and one beam (S8). $Z$ stands for the impurity ion charge and $m$ for the mass.

\begin{tabular}{llll}
\hline Parameter & Symbol & $2 \mathrm{NBIs}$ & $1 \mathrm{NBI}$ \\
\hline Magnetic field & $B_{\text {mod }}$ & $2.6 \pm 0.1 \mathrm{~T}$ & $2.6 \pm 0.1 \mathrm{~T}$ \\
Electron density & $n_{e}$ & $6.6 \pm 0.3 \cdot 10^{19} \mathrm{~m}^{-3}$ & $6.5 \pm 0.3 \cdot 10^{19} \mathrm{~m}^{-3}$ \\
Electron temperature & $T_{e}$ & $2.9 \pm 0.3 \mathrm{keV}$ & $2.2 \pm 0.3 \mathrm{keV}$ \\
Ion temperature & $T_{i}$ & $3.7 \pm 0.5 \mathrm{keV}$ & $2.1 \pm 0.5 \mathrm{keV}$ \\
Ion drift velocity & $V_{i}$ & $1.1 \pm 0.6 \cdot 10^{5} \mathrm{~m} / \mathrm{s}$ & $0.6 \pm 0.6 \cdot 10^{5} \mathrm{~m} / \mathrm{s}$ \\
Isotope ratio $n_{H} /\left(n_{H}+n_{D}\right)$ & $R_{i}$ & $0.03 \pm 0.02$ & $0.03 \pm 0.02$ \\
Impurity $(Z / m=6 / 12) n_{i 1} / n_{e}$ & $N_{i 1}$ & $0.005 \pm 0.005$ & $0.005 \pm 0.005$ \\
Impurity $(Z / m=50 / 184) n_{i 2} / n_{e}$ & $N_{i 2}$ & $0.0005 \pm 0.0005$ & $0.0005 \pm 0.0005$ \\
\hline
\end{tabular}

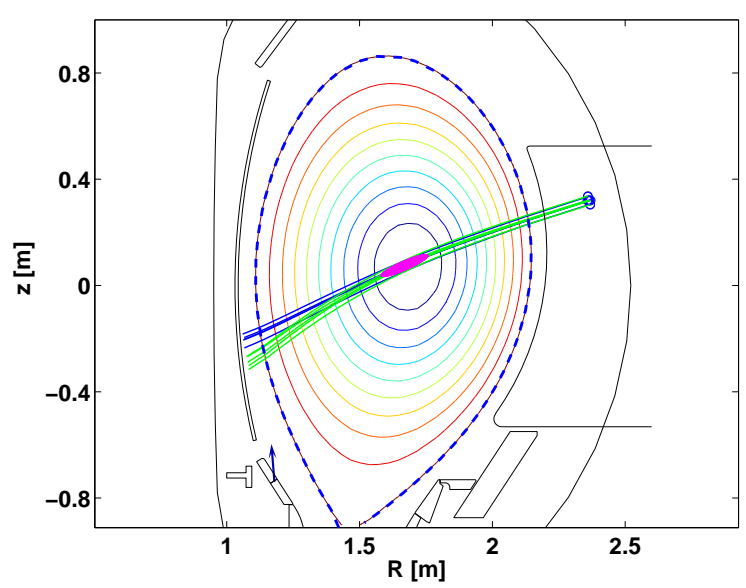

(a) Poloidal view

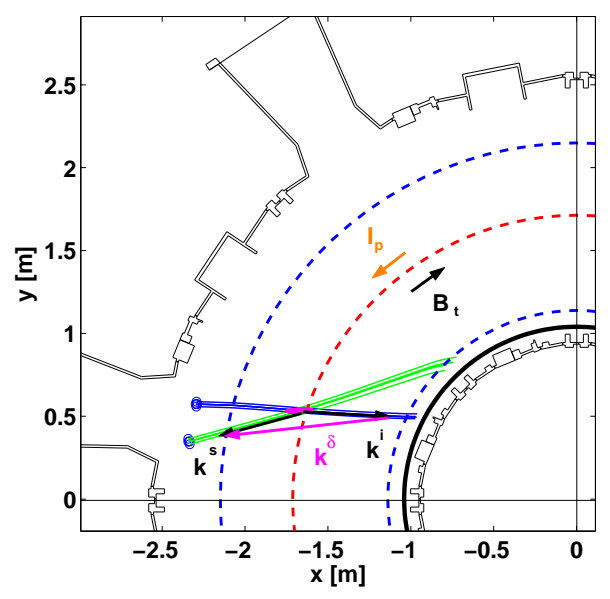

(b) Toroidal view

Figure 2. Ray tracing for the scattering geometry in discharge 24089 projected into the poloidal and toroidal planes, respectively. The probe beam is plotted in blue, the receiver beam in green. Both have O-mode polarization. The scattering volume lies at the intersection of probe and receiver beams and is illustrated in magenta. In Figure 2(b) viewing the torus from the top, the directions of the toroidal magnetic field $B_{t}$, the plasma current $I_{p}$ and the projected wave vectors $\mathbf{k}^{\delta}, \mathbf{k}^{i}$ and $\mathbf{k}^{s}$ are indicated. The dashed lines represent the magnetic axis (red) and the last closed flux surface (blue).

\subsection{CTS Experiment description}

In CTS experiments at ASDEX Upgrade, a probe beam of millimeter waves is launched into the plasma, and radiation is scattered off microscopic fluctuations in the plasma. Part of this scattered radiation is collected by a receiver with a quasi-optical antenna and resolved into frequency intervals. The acceptance cone of the antenna, referred to as the receiver beam, is defined by the antenna design. Information about the fast ions can be inferred from the spectral power density of the scattered radiation. The measurement is 
spatially resolved at the location where probe and receiver beam patterns intersect. This location is called the scattering volume and lies near the plasma center at $R=1.7 \mathrm{~m}$ and $z=0.1 \mathrm{~m}$ in this experiment. The scattering geometry is sketched in poloidal and toroidal views in Figures 2(a) and 2(b), respectively. CTS enables measuring the fast ion velocity distribution projected onto the fluctuation wave vector $\mathbf{k}^{\delta}=\mathbf{k}^{s}-\mathbf{k}^{i}$ where the superscripts $s$ and $i$ refer to scattered and incident radiation, respectively. The spectral content of the scattered radiation bears a signature of the ion velocity distribution since the CTS system at ASDEX Upgrade satisfies Salpeter's condition [51]: $\left|\lambda_{D} \mathbf{k}^{\delta}\right|<1$ where $\lambda_{D}$ is the Debye length. A frequency shift in scattered radiation, $\nu^{\delta}$, can be approximately related to an ion velocity, $\mathbf{v}_{\text {ion }}$, by $\nu^{\delta}=\nu^{s}-\nu^{i} \approx \mathbf{v}_{\text {ion }} \cdot \mathbf{k}^{\delta} / 2 \pi$.

In discharge 24089 the incident gyrotron power was $P_{i}=250 \mathrm{~kW}$ at an average frequency of $F_{i}=104.93 \mathrm{GHz}[25,26,52]$. The geometry and gyrotron parameters for the CTS experiment and their estimated uncertainties are summarized in Table 2 . The resolved angle $\phi=\angle\left(\mathbf{k}^{\delta}, \mathbf{B}\right)$, the scattering angle $\theta=\angle\left(\mathbf{k}^{i}, \mathbf{k}^{s}\right)$ and the less important azimuthal angle $\psi$ describe the scattering geometry. The so-called beam overlap $O_{b}$ accounts for the effects of the widths of the probe and receiver beam patterns and for the extent to which they overlap in the plasma $[38,39]$. The role of the overlap in our comparison is explained in Section 4.1. This set of CTS system parameters (Table 2) and the set of plasma parameters (Table 1) form the set of nuisance parameters mentioned in Section 2.1.

The magnetic field in discharge 24089 was $2.6 \mathrm{~T}$ on axis, placing the cold electron cyclotron resonances near the plasma edge so as to avoid large levels of electron cyclotron emission (ECE) background radiation and plasma heating. The ECE background is the largest contribution to CTS noise and amounts to several tens of eV against which a CTS signal on the order of $1-10 \mathrm{eV}$ has to be observed (for large frequency shift). The gyrotron is modulated in order to estimate the ECE background. The ECE background in the periods in which the gyrotron fires can then be estimated from the ECE background in the periods in which the gyrotron is off. The ECE background estimation turned out to be more cumbersome for ASDEX Upgrade plasmas compared to TEXTOR plasmas $[23,24]$ due to the fast time-scale MHD activity in typical ASDEX Upgrade plasmas, and a new approach to estimate the ECE background was adopted [26]. The ECE background is estimated using channels in which no CTS signal is expected. The uncertainty due to the ECE background subtraction was estimated by applying the formalism to plasmas without gyrotron operation. Here the estimated ECE background could be compared with the measured ECE background. The discrepancies between the estimated and measured ECE background were found to be smaller than the pulse-to-pulse variation of the measured CTS signal. In this work we present data which is averaged over 15 gyrotron pulses spanning $75 \mathrm{~ms}$, and the uncertainty of the measurement in each channel is given by the standard deviation of the time series. The radial extent of the scattering volume in this experiment was approximately $10 \mathrm{~cm}$ as illustrated in Figure 2. A more detailed description of the system parameters and the capabilities of CTS at ASDEX Upgrade can be found in references [25,26]. 
Table 2. CTS system parameters for the experiment in discharge 24089 and their estimated $1 \sigma$ confidence intervals. The orientations of the vectors $\mathbf{k}^{\delta}, \mathbf{k}^{i}, \mathbf{k}^{s}$, and $\mathbf{B}$ are sketched in Figure 2(b).

\begin{tabular}{lll}
\hline Parameter & Symbol & Value \\
\hline Resolved angle $\angle\left(\mathbf{k}^{\delta}, \mathbf{B}\right)$ & $\phi$ & $122 \pm 1^{\circ}$ \\
Scattering angle $\angle\left(\mathbf{k}^{i}, \mathbf{k}^{s}\right)$ & $\theta$ & $161 \pm 1^{\circ}$ \\
Azimuthal angle of $\mathbf{B}$ when $\hat{\mathbf{z}} \| \mathbf{k}^{\delta}$ and $\hat{\mathbf{x}} \|\left(\mathbf{k}^{i} \times \mathbf{k}^{s}\right)$ & $\psi$ & $-95 \pm 1^{\circ}$ \\
Gyrotron power & $P_{i}$ & $250 \pm 20 \mathrm{~kW}$ \\
Gyrotron frequency & $F_{i}$ & $104.93 \pm 0.01 \mathrm{GHz}$ \\
Beam overlap & $O_{b}$ & $70 \pm 301 / \mathrm{m}$ \\
\hline
\end{tabular}

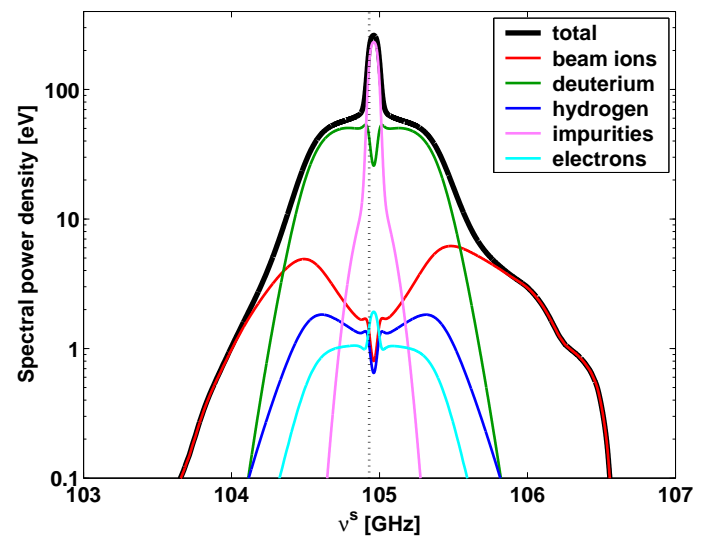

Figure 3. Synthetic CTS spectrum for the two-beam phase (S3+S8) in discharge 24089. The spectrum was calculated using the data from Tables 1 and 2 and the fast ion distribution simulated with TRANSP/NUBEAM shown in Figure 4(a). The total signal is the sum of the individual contributions indicated in this figure. The dotted line marks the gyrotron frequency.

\section{Methods for CTS data analysis}

Experimental CTS results can be compared with theoretical expectations in two complementary ways: either in frequency space, if a synthetic CTS spectrum is calculated from theoretical ion and electron velocity distributions, or in velocity space, if the one-dimensional fast ion velocity distribution $g(u)$ is inferred from the experimental CTS spectra. The velocity space comparison has the advantage that it is the velocity distribution $g(u)$ that one is actually interested in. On the other hand, the frequency space comparison has the advantage that the spectral power density is the quantity that is directly measured with the CTS receiver. The latter option is presented in Section 4.1 and the former in Section 4.2. We thus need to find the experimental and the simulated $g(u)$ and the experimental and the synthetic spectral power densities which are obtained by methods explained in the following three sections. 


\subsection{Forward model of CTS and synthetic spectra}

The forward model provides a mapping from velocity space to frequency space [38, 39]: if the velocity distributions of fast ions (see Section 3.2), bulk ions and electrons as well as the nuisance parameters are known, the expected CTS spectrum can be calculated $[31,53]$. Not only do calculated spectra rely on this forward model but also the estimation of $g(u)$ from the measured spectra (Section 3.3). The comparison of experimental and numerical data therefore also depends on the forward model. Predicted spectra are calculated using the best available estimates for the plasma and CTS system parameters (Tables 1 and 2). The spectral power density of the scattered radiation $\frac{\partial P^{s}}{\partial \nu^{s}}$ is calculated as

$$
\frac{\partial P^{s}}{\partial \nu^{s}}=P_{i} O_{b} n_{e} \lambda_{0}^{i} r_{e}^{2} \frac{1}{2 \pi} \Sigma
$$

where $\lambda_{0}^{i}$ is the vacuum wavelength of the probe radiation, $r_{e}$ the classical electron radius and $\Sigma$ the scattering function that gives the spectral shape [38,39]. This forward model is derived from a kinetic, fully electromagnetic description of the scattering process, and it accounts for fluctuations in electron density, in the electric and magnetic fields and in the electron current density.

Figure 3 shows a synthetic CTS spectrum calculated using this forward model for the NBI configuration with two beams $(\mathrm{S} 3+\mathrm{S} 8)$. The bulk ion species are assumed to consist of deuterium, hydrogen, carbon and tungsten with Maxwellian velocity distributions parameterized by a common bulk ion temperature and toroidal drift velocity with densities of the various ion species as given in Table 1 . However, the deuterium velocity distribution function is the sum of the beam ion velocity distribution function $g(u)$ (Section 3.2) and the Maxwellian distribution for the bulk deuterium. The electrons are assumed to have a Maxwellian distribution characterized by the electron temperature and density. The various species in the plasma each contribute a component to the CTS spectrum given by their assumed velocity distributions. The bulk ion feature dominates the spectrum from 104.3 - 105.5 GHz, the main contributions coming from deuterium and impurities. Note that the bulk ion feature has a small frequency shift from the gyrotron frequency due to the assumed bulk ion drift velocity. The beam ions are clearly the largest contributor to the CTS spectrum for frequencies above $105.5 \mathrm{GHz}$ and below $104.3 \mathrm{GHz}$ in this configuration. The asymmetry of the fast ion feature is a consequence of the asymmetry of the one-dimensional beam ion velocity distribution function to be discussed in Section 3.2.

\subsection{Simulation models for the fast ion velocity distributions}

In this section we describe how to obtain one-dimensional velocity distribution functions $g(u)$ from numerical simulation with help of the widely used transport code TRANSP coupled with the neutral beam module NUBEAM [40,41] or the test-particle Monte Carlo code ASCOT $[42,43]$. The simulation codes compute the two-dimensional fast ion

distribution $f\left(v_{\|}, v_{\perp}\right)$ at the location of the scattering volume where $v_{\|}$and $v_{\perp}$ refer to the 


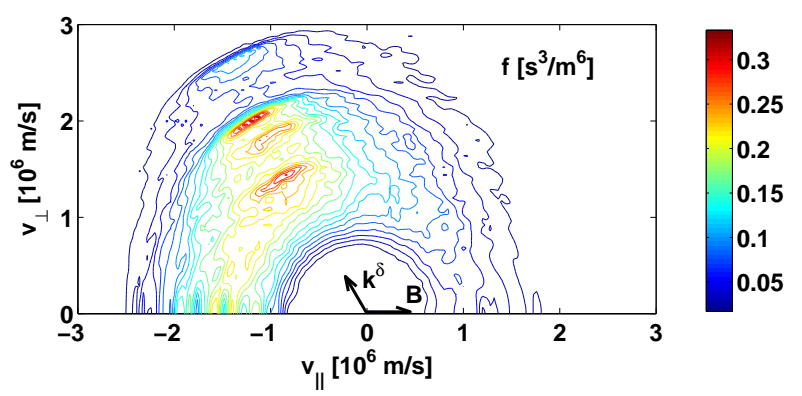

(a) TRANSP/NUBEAM

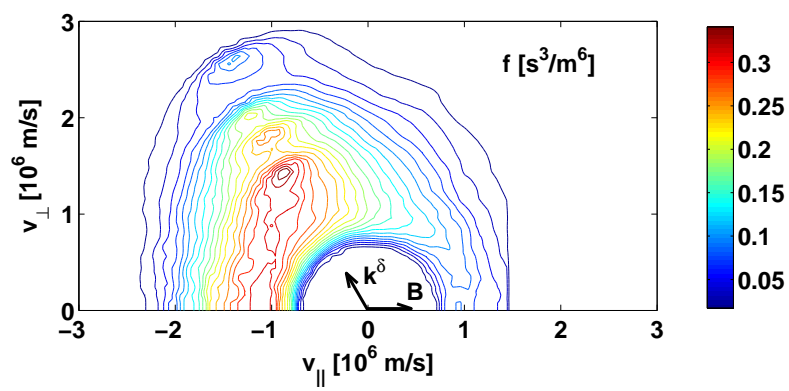

(b) ASCOT

Figure 4. Equally spaced contours of the beam ion velocity distribution $f$ in the scattering volume $(R=1.7 \mathrm{~m}, z=0.1 \mathrm{~m})$ computed with TRANSP/NUBEAM (a) and ASCOT (b) for heating with S3 and S8. The bulk ion distribution is not plotted in this figure, leading to low densities near the origin. The directions of the magnetic field $\mathbf{B}$ and $\mathbf{k}^{\delta}$ are indicated.

velocity components parallel and perpendicular to the magnetic field, respectively. We compare the CTS results with both sets of simulation results (from TRANSP/NUBEAM and from ASCOT). A full comparison of the two codes is, however, beyond the scope of this work. The steady-state velocity space distribution of beam ions originating from S3 and S8, as computed with ASCOT and TRANSP/NUBEAM, is presented in Figure 4. The largest velocity space densities for these beams are found at a pitch angle of $120^{\circ}$. The rotationally symmetric distribution $f\left(v_{\|}, v_{\perp}\right)$ is then projected onto the resolved direction $\mathbf{k}^{\delta}$ indicated in Figure 4 . The projection $g(u)$ of the full velocity distribution function $f\left(v_{\|}, v_{\perp}\right)$ along the direction of $\mathbf{k}^{\delta}$ is given by

$$
g(u)=\int d \mathbf{v} f \delta\left(\frac{\mathbf{v} \cdot \mathbf{k}^{\delta}}{k^{\delta}}-u\right)
$$

where $\delta()$ is the Dirac $\delta$-function and $u$ is the resolved one-dimensional velocity component.

We illustrate the projection of the rotationally invariant function $f$ onto $\mathbf{k}^{\delta}$ in Figure 5, using ASCOT data as example. The rotation axis $\mathbf{B}$ and $\mathbf{k}^{\delta}$ define a plane which slices through the three-dimensional, rotationally invariant function $f$. The resolved angle in the experiment was $\phi=\angle\left(\mathbf{k}^{\delta}, \mathbf{B}\right)=122^{\circ}$ indicated as the bold red line in Figure 5(a). Additionally, other options for resolved directions are shown for illustration: $\phi=20^{\circ}, \phi=90^{\circ}$, and $\phi=160^{\circ}$. Resolved angles of $\phi=20^{\circ}$ and 


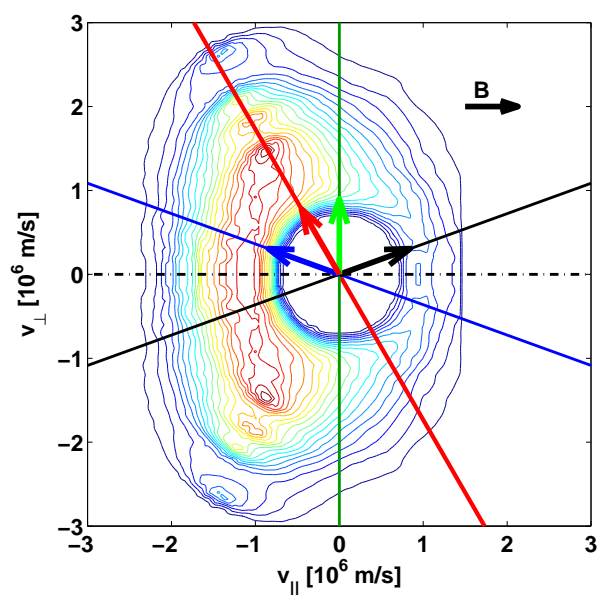

(a)

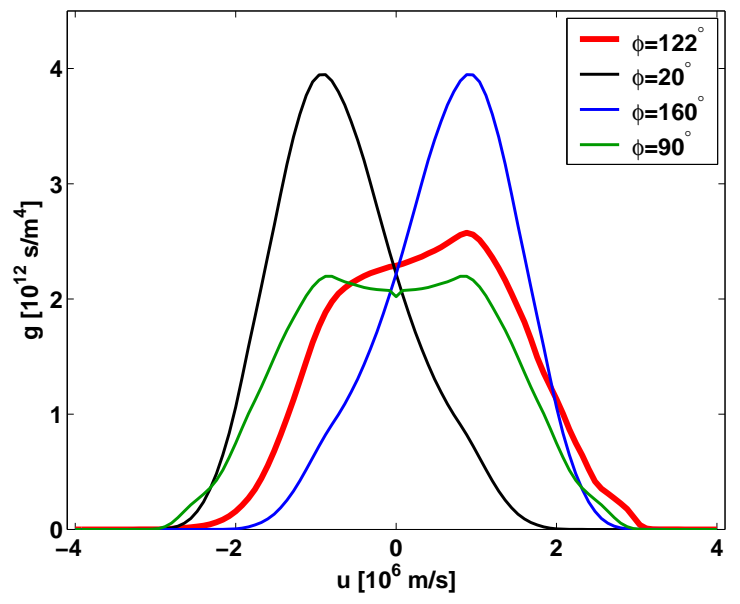

(b)

Figure 5. Illustration of the projection of the rotationally symmetric function $f$ from ASCOT onto $\mathbf{k}^{\delta}$. The straight colored lines represent various options for the resolved angle $\phi=\angle\left(\mathbf{k}^{\delta}, \mathbf{B}\right)$. The bold red line is at an angle of $\phi=122^{\circ}$ and is selected for this experiment. a) Slice through the rotationally symmetric fast ion distribution plotted in Figure 4(b). The plane contains $\mathbf{k}^{\delta}$ and the rotation axis B. b) Corresponding one-dimensional fast ion distribution functions $g(u)$ for various resolved angles $\phi$. The entire three-dimensional rotationally symmetric function $f$ is projected onto $\mathbf{k}^{\delta}$.

$\phi=160^{\circ}$ are at the limits of the steering capability of the CTS antenna system at ASDEX Upgrade. The corresponding projections $g(u)$ onto these resolved directions $\mathbf{k}^{\delta}$ are plotted in Figure 5(b). If the resolved direction is $\phi=90^{\circ}$, the projection of $f$ onto $\mathbf{k}^{\delta}$ will be symmetric about $u=0$. For resolution as close to parallel to the magnetic field as possible, $\phi=20^{\circ}$ or $\phi=160^{\circ}$, the projection becomes very asymmetric. These two projections are mirror images of each other. For the resolved angle chosen in this experiment, $\phi=122^{\circ}, g(u)$ is asymmetric about $u=0$ which leads to the asymmetry in the computed spectra described in Section 3.1. For this angle a finite phase space density exists at the largest values of the velocity component $u$. Scattering is then found at the largest possible frequency upshift with this angle. Thus the spectral range of the CTS receiver for frequency upshift is exploited optimally for this angle.

\subsection{Inference of the fast ion velocity distribution from CTS measurements}

The inference of the one-dimensional fast ion velocity distribution $g(u)$ and of its estimated uncertainty - the parameters of interest - demands the solution to an inverse problem. Direct operators mapping from frequency space (the measured CTS spectrum) to velocity space are not available whereas a forward model mapping from velocity space to frequency space has been formulated (Section 3.1) $[38,39]$. The velocity distribution $g(u)$ is obtained from the measured spectra by a least square fitting procedure using this forward model. Uncertainties in the nuisance parameters and in the measured spectral power density are accounted for. Assuming normal distributions for the measured spectral power densities and the nuisance parameters, one can derive expressions for the 


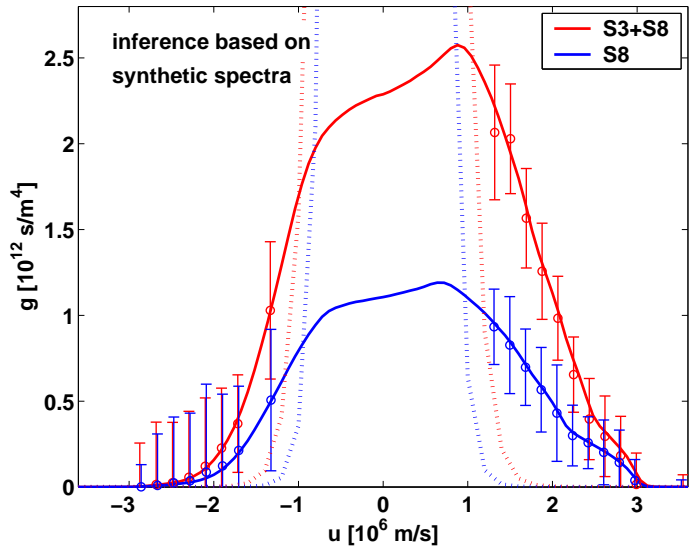

Figure 6. Comparison of the simulated one-dimensional fast ion velocity distributions $g(u)$ and the inference from synthetic spectra based on these simulated distributions. The plasma is assumed to be heated by two beams (S3+S8, red) and one beam ( 8 , blue); - ASCOT, o - inference based on synthetic spectrum, $\cdots$ - bulk ions. We plot the inferred fast ion distributions for velocities larger than typical for the bulk ions.

misfit between the experimental data and the expectation [54]. The nuisance parameters and their uncertainties are summarized in Tables 1 and 2.

We demonstrate the inference in Figure 6 for the simulated one-dimensional fast ion distribution function $g(u)$. A synthetic spectrum based on this distribution function and the nuisance parameters in Tables 1 and 2 was displayed in Figure 3. We infer $g(u)$, given only the synthetic spectrum and the nuisance parameters as well as their uncertainties. We assume the uncertainties of the synthetic spectrum to be given by the measurement uncertainties of the actual experiment. In the inference we make use of the full spectrum, except for the parts which are blocked by the notch filter in the experiment. In this way the inference is demonstrated for experimental conditions. The inference should then ideally be identical to the fast ion distribution function used to calculate the synthetic spectrum. Figure 6 demonstrates that the underlying fast ion distributions can be inferred from the synthetic spectra with good accuracy.

\section{Results}

\subsection{Comparison of measured and synthetic CTS spectra}

Figure 7 presents a comparison of experimentally obtained spectra against synthetically generated spectra, such as the one shown in Figure 3, for both NBI configurations (S3+S8 and S8 only). The synthetic spectra obtained on the basis of TRANSP/NUBEAM or ASCOT simulations agree reasonably well with each other, especially for large frequency shifts. We note several points of agreement between the simulations and the measurements. First, there is a clear asymmetry about the gyrotron frequency due to the presence of beam ions in the measurement and in the simulation, the reason for which we explained in Sections 3.1 and 3.2. Second, the larger particle 


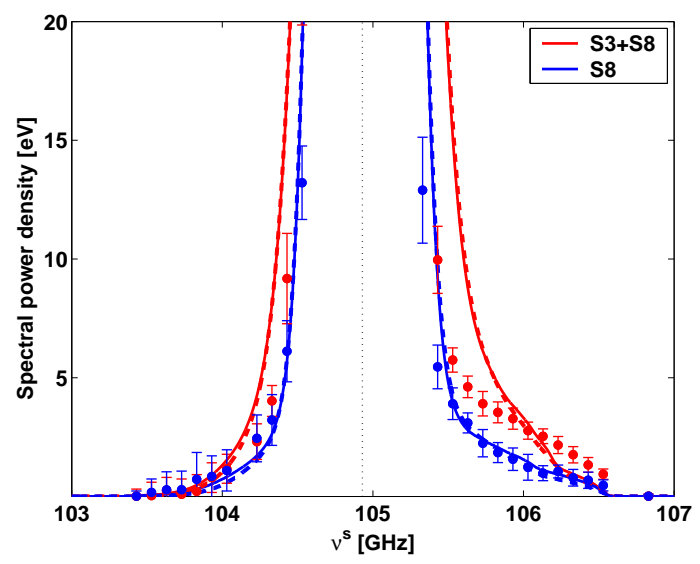

Figure 7. Comparison of measured and synthetic CTS spectra for discharge 24089 for auxiliary heating with two NBI sources (S3+S8, red) and one NBI source (S8, blue); TRANSP/NUBEAM, - - - ASCOT, o- measurement, $\cdots$ - gyrotron frequency; CTS measurements were averaged over 15 gyrotron pulses. The two-beam configuration is also presented in logarithmic scale in Figure 8 as reference.

density of beam ions in the two-beam phase $(\mathrm{S} 3+\mathrm{S} 8)$ is reflected in the larger spectral power densities for frequencies above $105.5 \mathrm{GHz}$. When neutral beam S3 is switched off, the spectral power density above $105.5 \mathrm{GHz}$ drops significantly compared to the error bars. We attribute this to a smaller population of fast ions (see Section 4.2). Measurements and simulations agree in this respect. Third, the bulk ion feature from $104.3-105.5 \mathrm{GHz}$ is measured to be narrower due to the ion temperature drop after $2.5 \mathrm{~s}$ as was expected according to simulation.

However, there are quantitative discrepancies between the simulation and the experiment. To point these out, we need to address the sensitivity of the synthetic spectra to changes in plasma parameters affecting the spectra. The sensitivity of the spectra to changes in a selection of such plasma parameters is shown in Figure 8 for the two-beam phase together with experimental data. Each parameter is varied while the other parameters are kept constant. Figure 8(a) shows that changes in the fast ion density affect mainly large frequency shifts beyond the bulk ion feature. Contrarily, the ion temperature has an impact on the width of the bulk ion feature and has only a small influence on the spectral power density for large frequency shifts (Figure 8(b)). These two effects were observed in Figure 7. Another important issue is the uncertainty due to an incompletely known frequency independent scaling factor. Equation (1) asserts that the measured spectral power density is directly proportional to the probing power $P_{i}$ and the beam overlap $O_{b}$ (the electron density $n_{e}$ also appears in the scattering function $\Sigma)$. The uncertainties in the probing power and the beam overlap can be combined with the uncertainty in the receiver calibration and have the effect of a frequency independent scaling factor. We show the sensitivities to the electron density and this scaling factor in Figures 8(c) and 8(d), respectively.

An uncertainty of a factor two in this scaling factor seems possible due to 
mainly systematic uncertainties whereas the stochastic error due to launcher control is significantly smaller (accuracy $<0.1^{\circ}$ ). One source of such systematic uncertainty is the antenna characteristic of the receiver beam which is only known for the component of radiation which excites an HE11-mode in the transmission line. Another systematic uncertainty is due to beam diffraction by transverse density gradients. A third systematic uncertainty is due to the power measurement of the probe beam using the embedded directional coupler which may not be accurate for short pulses. A direct calorimetric measurement of a short sequence of these pulses is in principle possible and should reduce this error component significantly $(<5 \%)$. An additional source of uncertainty lies in the calibration of the CTS receiver.

Due to the relatively large uncertainties in the scaling factor illustrated in Figure 8(d), one should only consider the shape of a CTS spectrum when comparing it to a simulation and allow an overall multiplicative factor. Such a frequency independent scaling factor will move the spectrum up or down in the logarithmic plot and scale the values and the gradients in a linear plot. In Figure 7 we use the nominal values as the best guess before consideration of the CTS data. Note that any deviation from these nominal values should be the same for the cases with one and with two beam sources shown in Figure 7 since the antenna setting is unchanged, the density profiles are very similar, and the nominal ECRH power is identical. This means that there is essentially only one common scaling factor to be applied to the simulated data. We will come back to this point in the discussion of Figure 10 at the end of Section 4.2. We do not comment on the sensitivities to other nuisance parameters further here even though these also have a bearing on the uncertainty of the measurement results [54]. An additional uncertainty originates from the finite size of the scattering volume which implies a distribution of $\mathbf{k}^{\delta}$. In the experiment the CTS signal will be measured for a convolution over the distribution of $\mathbf{k}^{\delta}$ whereas only the nominal $\mathbf{k}^{\delta}$ is assumed in our model. Moreover, we note that the nuisance parameters may not be constant within our scattering volume. However, these uncertainties can be shown to be small compared with the uncertainty due to uncertain nominal values of the nuisance parameters.

For the discussion of Figure 7 we keep in mind that the simulation results are uncertain with respect to an overall scaling factor between 0.5 and 2. We note that the computation predicts a significantly steeper gradient in the spectra for the two-NBI configuration (S3+S8) compared with the one-NBI configuration (S8). This difference in gradient is smaller in the measured spectra. A second difference is that the measured bulk ion feature has a tendency to be narrower than the expected bulk ion feature as is also visible in Figure 8. It can furthermore be noted that the expected decrease in spectral power densities, after S3 is switched off, for negative frequency shift is too small to be measured. The error bars in this frequency range (below $104.3 \mathrm{GHz}$ ) are larger due to the higher ECE background levels for these frequencies in this experiment. 


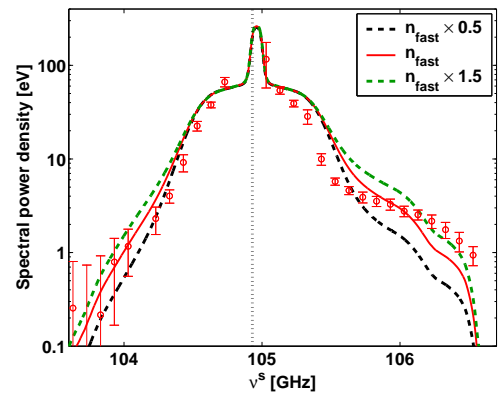

(a) Fast ion density

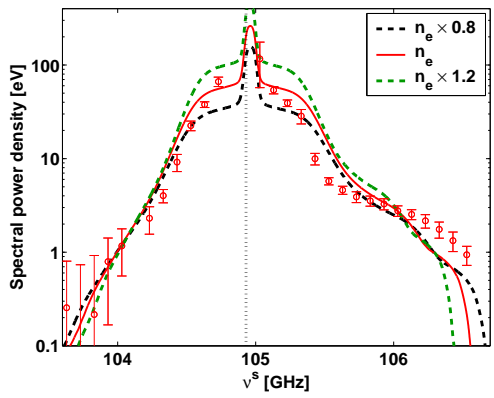

(c) Electron density

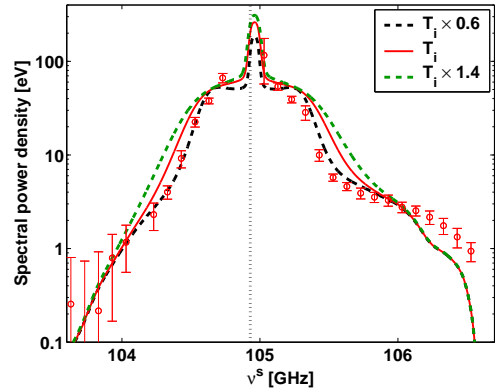

(b) Ion temperature

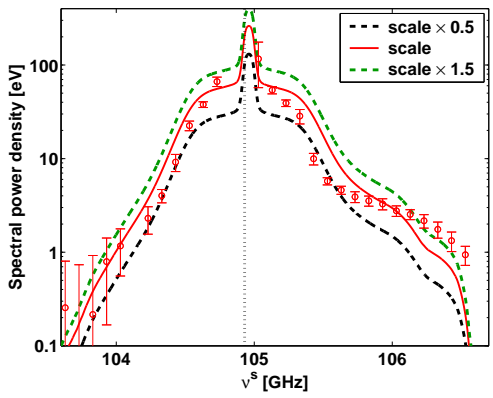

(d) Scaling factor

Figure 8. Sensitivity of the spectra to changes in the fast ion density, the ion temperature, the electron density and the frequency independent scaling factor for the two-NBI configuration. The spectra are here presented in logarithmic scale. The baseline spectrum (red) was also displayed in linear scale in Figure 7. One parameter is varied in each figure as indicated. The fast ion velocity distribution function has not been recomputed in each case.

\subsection{Comparison of measured and simulated fast ion velocity distributions}

In Figure 9 we present the inferred fast ion distributions from the CTS measurements and compare them with the simulated fast ion distribution from TRANSP/NUBEAM or ASCOT. Also in velocity space the simulations and experiments agree in several points. First, the projection of the beam ion distribution function onto the resolved direction is asymmetric: the measured $g(u)$ is larger for positive $u$ compared with negative $u$ which was expected from the simulations. Second, $g(u)$ decreases for positive $u$ when neutral beam S3 is turned off as expected. This decrease is only evident for positive $u$ whereas $g(u)$ for both NBI configurations coincides for negative $u$ within the asserted confidence. The different shape of $g(u)$ for each NBI configuration can be clearly observed and originates from the different beam energies. The asymmetry is more pronounced in the two-beam configuration. There are also some discrepancies: there is a tendency that the simulated fast ion velocity distributions lie below the measured ones. Furthermore, the difference in the gradients between the two-beam and one-beam phases for positive $u$ is larger in the computations than in the CTS measurements. The causes of these tendencies are presently under investigation.

As noted before, the projections $g(u)$ of the simulation results from 


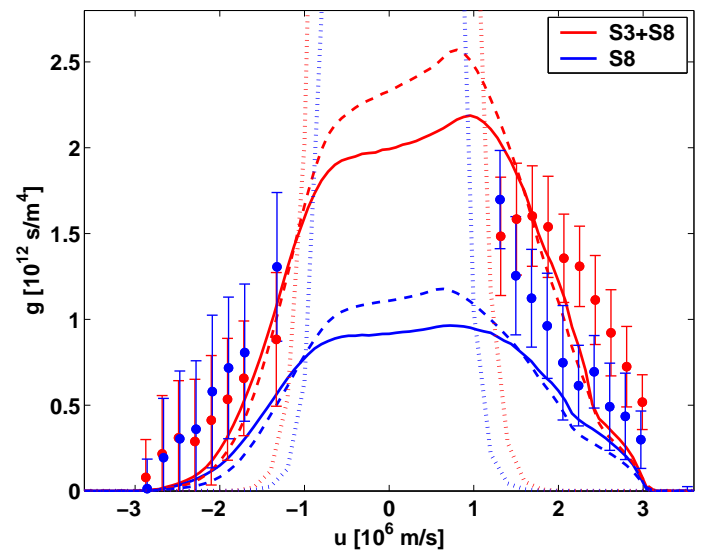

Figure 9. Comparison of the measured and computed one-dimensional fast ion velocity distributions $g(u)$ for plasma heating by two beams (S3+S8, red) and one beam (S8, blue); - TRANSP/NUBEAM, - - - ASCOT, o - measurement, $\cdots$ - bulk ions. The measured distribution is restricted to outside indicated bulk ion distributions. The beam ion energies $60 \mathrm{keV}$ and $93 \mathrm{keV}$ correspond to $2.4 \cdot 10^{6} \mathrm{~m} / \mathrm{s}$ and $3 \cdot 10^{6} \mathrm{~m} / \mathrm{s}$ for $\angle\left(\mathbf{v}_{i o n}, \mathbf{k}^{\delta}\right)=0$, respectively.

TRANSP/NUBEAM and ASCOT onto $\mathbf{k}^{\delta}$ are very similar for large $u$ (see Figure 9). They cannot be told apart with the CTS diagnostic in this experiment as the discrepancies between TRANSP/NUBEAM and ASCOT are below the uncertainty of the experimental results for large $u$. We stress that small velocity components $u$ are not necessarily related to small ion velocities: the $\delta$-function in Equation (2) picks out $u=\mathbf{v} \cdot \mathbf{k}^{\delta} / k^{\delta}$, and so $u$ will be small even for large ion velocities $\mathbf{v}$ if $\mathbf{v}$ and $\mathbf{k}^{\delta} \operatorname{span}$ a large angle. The bulk ions mask this fast ion information for small $u$ components. Therefore, the inferred fast ion velocity distribution is plotted only for velocities beyond the Maxwellian bulk indicated in Figure 9. The error bars show the $1 \sigma$ confidence interval for the phase space density of fast ions at each velocity. They are affected by the measurement uncertainties of the spectral power density and also those of the nuisance parameters. The uncertainties in the velocity distribution are correlated and are mostly such that they could be represented by uncertainty in a scaling factor for the velocity distribution. It can be shown to mostly originate from the uncertain frequency independent scaling factor of the spectral power density discussed in Section 4.1 in combination with other uncertainties in the nuisance parameters [54]. Considering the uncertainties of both theory and experiment, these first comparisons of CTS results to numerical plasma simulations show a reasonable level of agreement.

Finally, we revise our comparison between the simulations and the experiments in frequency space after consideration of the measured spectral power densities. The comparison before consideration of the measured spectral power densities was shown in Figure 7 which was therefore a prediction of the signal to be observed. Here we pick the comparison of the measurement with NUBEAM data and compare the prediction before the experiment with the newly computed maximum likelihood estimate. The maximum likelihood solution for all parameters also contains revised estimates for the nuisance 


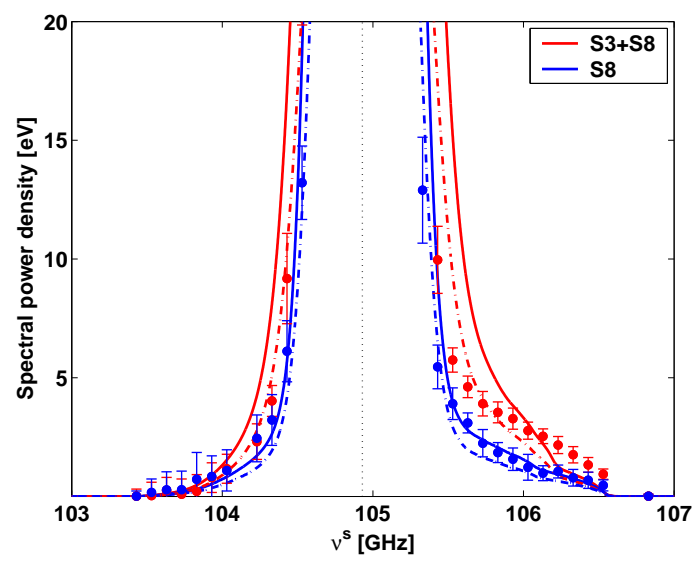

Figure 10. Comparison of measured and synthetic CTS spectra for discharge 24089 for auxiliary heating with two NBI sources (S3+S8, red) and one NBI source (S8, blue); TRANSP/NUBEAM before consideration of the CTS data, - - - TRANSP/NUBEAM after consideration of the CTS data consistent with the corresponding $g(u)$ from Figure 9, o - measurement, $\cdots$ - gyrotron frequency.

parameters, in particular the magnitude of the frequency independent scaling factor. The maximum likelihood solution suggests this factor to be smaller than estimated originally. The measured spectral power densities for small frequency shifts are lower than the predicted values which leads to a downward revision of the magnitude of the frequency independent scaling factor by $30 \%$. Therefore, the simulated levels of spectral power densities are overall lower after consideration of the CTS data as shown in Figure 10. This downward revision was also illustrated in Figure 8(d). We note that the scaling factors determined with the maximum likelihood solution are very similar

for both NBI configurations as expected from the discussion on the nature of the scaling uncertainty in section 4.1. Thus we reconciled the comparison in frequency space with our maximum likelihood solution of the one-dimensional velocity distribution.

\section{Discussion}

We have noted some points of agreement and some points of disagreement between measurements and simulations. We observe agreement among the ASCOT and TRANSP/NUBEAM simulation codes in experimentally accessible parameters. However, it may be possible to find a heating scenario for which the two codes make different predictions which could be experimentally distinguishable by CTS. This will require some benchmarking efforts among the codes and additional CTS experiments which is beyond the scope of the present work. In future experiments the results of simulations could be compared with CTS measurements for several locations in the plasma and several resolved directions for various heating scenarios. Furthermore, it will be feasible to reduce the uncertainties of the CTS measurements. Recent modifications of the CTS receiver will allow the spectral power density to be measured with higher accuracy. Additionally, CTS data analysis will benefit from more accurate measurements 
of the nuisance parameters. In the present work the full capabilities of the CTS system have not yet been fully exploited: the signal-to-noise ratio has been increased at the expense of time resolution. In future experiments it may be possible to inject higher gyrotron powers [52] which may improve the signal-to-noise ratio and thus enable us to increase the time resolution. This may allow detailed studies of plasma dynamics on millisecond time scales by CTS - the results of which may be interesting to compare with numerical simulations. It would also be of great benefit to add a second receiver to the CTS system at ASDEX Upgrade. A second receiver located in a different port would enable CTS measurements resolving simultaneously two directions and would allow a reconstruction of two-dimensional fast ion velocity distribution functions [55].

\section{Conclusions}

The fast ion populations in the plasma center of ASDEX Upgrade were compared for two NBI configurations: one neutral beam versus two neutral beams. One-dimensional fast ion velocity distributions $g(u)$ were inferred for both NBI configurations. The inferred $g(u)$ and the measured CTS spectra for both heating regimes have different shapes and can, as expected, clearly be distinguished. The CTS measurements were compared with simulations using the TRANSP/NUBEAM and ASCOT codes. Salient features of the measured spectral power densities and one-dimensional fast ion velocity distributions are in reasonable agreement with the simulations within the limits of the given uncertainties. Quantitative discrepancies between measurement and simulation in absolute values and gradients were observed which will drive future activities in the development of the diagnostic and the codes.

\section{Acknowledgments}

This work, supported by the European Communities under the contract of Association between EURATOM and Risø DTU was partly carried out within the framework of the European Fusion Development Agreement. The views and opinions expressed herein do not necessarily reflect those of the European Commission. The computing facilities of the Finnish IT Center for Science (CSC) used in this work are thankfully acknowledged. This work was partially funded by The Finnish Academy project No. 121371.

\section{References}

[1] Herrmann A and Gruber O 2003 Fusion Sci. Tech. 44 569-577

[2] Gruber O et al 2007 Nucl. Fusion 47 S622-S634

[3] Chen L 1994 Phys. Plasma 1 1519-1522

[4] Briguglio S et al 2007 Phys. Plasma 14 055904-055904-10

[5] Heidbrink W W and Sadler G J 1994 Nucl. Fusion 34535

[6] Zweben S J et al 2000 Nucl. Fusion 4091 - 149

[7] Heidbrink W W 2002 Phys. Plasma 92113 - 2119

[8] Pinches S D et al 2004 Plasma Phys. Control. Fusion 46 B187-B200 
[9] Campbell D J et al 1988 Phys. Rev. Lett. 60 2148-2151

[10] Marcus F B et al 1994 Nucl. Fusion 34(5) 687-701

[11] Kolesnichenko Y I, Lutsenko V V, White R B and Yakovenko Y V 2000 Nucl. Fusion 40 1325-1341

[12] Graves J et al 2005 Plasma Phys. Control. Fusion 47 B121-B133

[13] Chang Z et al 1996 Phys. Rev. Lett. 761071

[14] Nazikian R et al 1996 Phys. Plasma 3(2) 593-605

[15] García-Muñoz M et al 2007 Nuclear Fusion 47 L10-L15

[16] García-Muñoz M et al 2008 Physical Review Letters 100055005

[17] Günter S et al 2007 Nucl. Fusion 47 920-928

[18] Hauff T, Pueschel M J, Dannert T and Jenko F 2009 Phys. Rev. Lett. 102075004

[19] Heidbrink W W et al 2009 Phys. Rev. Lett. 103175001

[20] ITER Physics Expert Group on Energetic Particles, Heating and Current Drive 1999 Nucl. Fusion 39 2471-2495

[21] Fasoli A et al 2007 Nucl. Fusion 47 S264-S284

[22] Bindslev H et al 1999 Phys. Rev. Lett. 83(16) 3206-3209

[23] Bindslev $\mathrm{H}$ et al 2006 Phys. Rev. Lett. 97205005

[24] Nielsen S K et al 2008 Phys. Rev. E 77016407

[25] Meo F et al 2008 Rev. Sci. Instrum. 79 10E501

[26] Meo F et al accepted J. Phys.: Conf. Series First results and analysis of collective Thomson scattering (CTS) fast ion distribution measurements on ASDEX Upgrade, presented at 14th International Symposium on Laser-Aided Plasma Diagnostics

[27] Bindslev H et al 2004 Rev. Sci. Instrum. 75(10) 3598-3600

[28] Meo F et al 2004 Rev. Sci. Instrum. 75 3585-3588

[29] Korsholm S B et al 2008 Burning Plasma Diagnostics (AIP conference proceedings vol 988) pp $118-122$

[30] Salewski M et al 2008 Rev. Sci. Instrum. 79(10) 10E729

[31] Salewski M et al 2009 Plasma Phys. Control. Fusion 51035006

[32] Leipold F et al 2009 Rev. Sci. Instrum. 80093501

[33] Machuzak J et al 1997 Rev. Sci. Instrum. 68 458-461

[34] Tartari U et al 2006 Nucl. Fusion 46928

[35] Westerhof E et al 2009 Phys. Rev. Lett. 103125001

[36] Nishiura M et al 2008 Rev. Sci. Instrum. 79(10) 10E731

[37] Suvorov E and et al 1995 Plasma Phys. Control. Fusion 371207

[38] Bindslev H 1993 Plasma Phys. Control. Fusion 35(11) 1615-1640

[39] Bindslev H 1996 J. Atmos. Terr. Phys. 58983

[40] Budny R V et al 1995 Nucl. Fusion 351497

[41] Pankin A, McCune D, Andre R and Kritz A 2004 Comp. Phys. Communications 159 157-184

[42] Heikkinen J A and Sipilä S K 1995 Phys. Plasma 23724 - 3733

[43] Heikkinen J A et al 2001 J. Comp. Phys. $173527-548$

[44] Streibl B et al 2003 Fusion Sci. Tech. 44 578-592

[45] Murmann H et al 1992 Rev. Sci. Instrum. 63 4941-4943

[46] Fischer R, Dinklage A and Pasch E 2003 Plasma Phys. Control. Fusion 45 1095-1111

[47] Fischer R et al 2008 Plasma Phys. Control. Fusion 50085009

[48] Fischer R et al 2008 Europhysics Conference Abstracts 32D P4.010 - Integrated Density Profile Analysis in ASDEX Upgrade H-modes

[49] Fischer R et al 2009 Europhysics Conference Abstracts 33E P1.159 - Multiple diagnostic data analysis of density and temperature profiles in ASDEX Upgrade

[50] Dux R 2003 Fusion Sci. Tech. 44 708-715

[51] Salpeter E E 1960 Phys. Rev. 120(5) 1528-1535

[52] Wagner D H et al 2008 IEEE Trans. Plasma Sci. 36 324-331

[53] Salewski M et al 2009 Nucl. Fusion 49025006 
[54] Bindslev H 1999 Rev. Sci. Instrum. 70 1093-1099

[55] Egedal J and Bindslev H 2004 Phys. Plasma 11(5) 2191-2198 